\title{
ENVIRONMENTAL IMPACT OF DYEING AND FINISHING CONVENTIONAL AND ORGANIC EGYPTIAN COTTON
}

\author{
Elkadi, M. Y. ${ }^{(1)}$; Ali, S. G..$^{(2)}$ and Zaki, M. A. ${ }^{(3)}$ \\ 1) Faculty of Science, Ain Shams University. 2) Dyeing and Finishing. Co. \\ 3) Textile Technology Center, Faculty of Engineering, Cairo University .
}

\begin{abstract}
The textile environmental issues associated with impact represent both cotton agriculture and dyeing processes . Cotton farms emit hazard chemical compounds as Piperonyl butoxide , Cypermethrin, Lambds- cyhalothrin, Chlorpyrifos, Profenofos, Malathion, Lufenuron and Carbendazim. These compounds were detected by multiresidue method analysis for convention cotton in addition to wet processes and dyeing operations ; high BOD,COD,TS, alkaline wastewater and wasted residual unfixed dyes. So this investigation focuses on monitoring of environmental contaminants and ecoproduction environmental friendly clothes as model which are more healthy for humans and safe on the ecosystem. On using organic cotton instead of conventional cotton, where organic agriculture farms there are no synthetic chemical pesticides, no synthetic fertilizers .The ring spun yarns of 30/1 Ne combed organic and conventional cotton were produced with similar parameters like beating point and settings. Rib knitted fabrics were produced with similar loop length and weight using organic and conventional cotton yarn. The fabrics were processed in eco textile processing by using Levafix Red CA Gran(vinyl sulphone and difluoropyrimidine) reactive dyes by soft flow machine in single dye bath. The fabrics were tested for colour fastness to washing, water, rubbing, perspiration, light and ecological emission measurements.
\end{abstract}

Keywords: Organic Egyptian cotton, Reactive dye behavior, Residual pesticides in cotton, Ecofreindly apparel. 
J. Environ. Sci.

Institute of Environmental Studies and Research - AinShamsUniversity

\section{INTRODUCTION}

In the textile industry, ecology is the most important aspect in the present worldwide scenario. $25 \%$ of the world's chemical pesticides are sprayed on pure cotton. Pesticides pollute our land, air, as well as, water. The toxic chemicals present in pure cotton could well be assimilated through our skin.

In the conventional cotton industry, pesticides are sprayed over the cotton crops, causing serious health problems to cotton workers and soil degradation ( Ferrigno, 2005). Organic production systems replenish and maintain soil fertility, reduce the use of toxic and persistent pesticides and fertilizers, and build biologically diverse agriculture. Third-party certification organizations verify that organic producers use only methods and materials allowed in organic production (Punj, 2000).Organic cotton is a crop that is grown without the use of synthetic chemicals such as pesticides, herbicides and fertilizers using methods and materials that have

a low impact on the environment. Cotton was always cultivated organically, like all crops, until the early 20th century, but the demand for cosmetically perfect production and higher yields gave rise to increase the use of synthetic pesticides and fertilizers, and subsequently to genetically modified cotton (Rieple A and Singh R; 2010). Consequently, this led to the accreditation and certification of organic production (P Ton 2002).

Organic cotton will have a smaller impact on Mother Nature from sustainable agriculture policies, as well as, processing without the utilization of insecticides and chemicals. 
Also, up to 200,000 tons of dyes are lost to effluents every year during the dyeing and finishing operations, due to the inefficiency of the dyeing processes. Unfortunately, most of these dyes need conventional wastewater treatment processes and persist in the environment as a result of their high stability to light, temperature, water, detergents, chemicals, soap and other parameters such as bleach and perspiration (Couto SR ;2009). The wastewater composition is depending on the different organic-based compounds, inorganic chemicals and dyes used in the industrial dry and wet-processing steps. Textile effluents from the dyeing and rinsing steps represent the most coloured fraction of textile wastewaters, and are characterized by extreme fluctuations in many quality indicators such as $\mathrm{COD}, \mathrm{BOD}, \mathrm{pH}$, colour, salinity and temperature.

Design approaches for the environment have evolved in scope and depth, beginning with environmental awareness and leading to a complex understanding of how society and the environment interact with and affect each other. Environmental design approaches started with green design, a term originally borrowed from the environmental "buzzword" of 1980's politics (Madge, 1997).

The present study aims to produce ecofreindly clothes with low environmental impacts starting from sourcing to readymade garments including safe textile chemistry science and dyeing technology by using organic cotton as cleaner product instead of conventional cotton. Also, hazardless chemical substances and nontoxic materials; are atilized to preserve the environment surrounding. 


\section{MATERIALS AND METHODS}

2.1. Fiber Measurements: Measurements of length parameters, tenacity properties and micronaire value for two samples from conventional and organic Egyptian cotton fibers; both of them are Giza 86.which related to HVI system according to ASTM D- 4603-86-1776-1998.(ASTM, 1998) are performed.

Table 1. Micronaire measurements

\begin{tabular}{|c|c|c|c|c|c|}
\hline \multicolumn{3}{|c|}{ Conventional cotton } & \multicolumn{3}{c|}{ Organic cotton } \\
\hline \hline MIC & MV & LD(mtex) & MIC & MV & LD (mtex) \\
\hline 3.87 & 0.80 & 176.00 & 4.16 & 0.87 & 179.00 \\
\hline \hline
\end{tabular}

All fibers were measured under atmospheric conditions at $65 \% \pm 2 \% \mathrm{Rh}$ and $21{ }^{\circ} \mathrm{C} \pm 2{ }^{\mathrm{O}} \mathrm{C}$ according to ASTM D1776- 2004. (ASTM, 2004).

2.2. Yarn samples: Conventional cotton yarn samples of $30 / 1$ and organic cotton 30/1 were spun in super spinning mill, Coimbatore with the following machines and process parameters.

2.3. Fabric samples: Rib conventional cotton fabric produced from conventional cotton yarn samples 30/1 $\mathrm{Ne}$ and also Rib organic cotton produced from Organic cotton yarn samples 30/1 Ne by Circular Knitting Machine 
Table 2 . Yarn and Fabric Process parameters.

\begin{tabular}{|c|c|c|}
\hline Structure of fabric & Conventional cotton & Organic cotton \\
\hline \hline Yarn Count & $30 / 1 \mathrm{Ne}$ & $30 / 1 \mathrm{Ne}$ \\
\hline Strength of yarn & $3.5 \mathrm{per}$ inch & $3.5 \mathrm{per}$ inch \\
\hline Number of columns & $\begin{array}{c}\text { Force }: 0.78 \mathrm{~kg} \\
\text { Elong. }: 11.5 \mathrm{~mm}\end{array}$ & $\begin{array}{c}\text { Force }: 0.815 \mathrm{~kg} \\
\text { Elong.: } 13.2 \mathrm{~mm}\end{array}$ \\
\hline Weight /squ.meter & 11 & 11 \\
\hline weight after dyeing & $175 \mathrm{~g} / \mathrm{m} 2$ & $175 \mathrm{~g} / \mathrm{m} 2$ \\
\hline Width of raw fabric & $200 \mathrm{~g} / \mathrm{m} 2$ & $200 \mathrm{~g} / \mathrm{m} 2$ \\
\hline Width after finish & $105 \mathrm{~cm}$ & $105 \mathrm{~cm}$ \\
\hline Gauge & $93 \mathrm{~cm}$ & $93 \mathrm{~cm}$ \\
\hline Diameter & $18 ”$ & $18 ”$ \\
\hline Circular Knitting M. & Orizio gauge 18 & Orizio gauge 18 \\
\hline
\end{tabular}

2.4. Scouring and dyeing: Scouring applies in overflow sample machine for conventional and organic fabrics then dyeing process was carried out with Levafix Red CA Gran (vinyl sulphone and difluoropyrimidine) reactive dyes, using exhaustion method (Figure. 1).

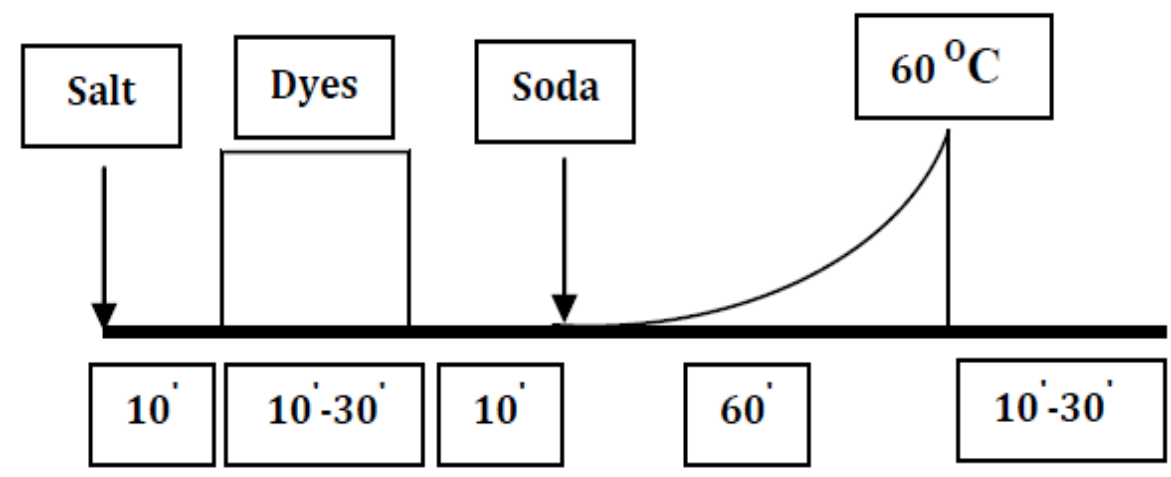

Figure 1. Dyeing Isothermal process. (Exhaustion method). 
Table 3. Scouring and dyeing processes parameters.

\begin{tabular}{|c|c|}
\hline \multicolumn{2}{|c|}{ Scouring parameters } \\
\hline Fabric weight & $\begin{array}{c}2 \mathrm{~kg} \text { conventional cotton } \& \\
2 \mathrm{~kg} \text { organic cotton }\end{array}$ \\
\hline Alkali $(\mathrm{NaOH})$ & $4 \mathrm{gm}$ per liter. \\
\hline Soda ash & 7 gm per liter PH 10.5 \\
\hline Wetting agent & 1 gm per liter. \\
\hline Sequestering agent & $1 \mathrm{gm}$ per liter \\
\hline Temperature & 100 to $125 \square \mathrm{c}$. \\
\hline Time & 1 hour (open vessel overflow) \\
\hline $\mathrm{M}: \mathrm{L}$ & $1: 10$ \\
\hline \multicolumn{2}{|c|}{ Dyeing parameters } \\
\hline Machine capacity & $1 \mathrm{~kg}$ to $5 \mathrm{~kg}$ \\
\hline Levafix Red CA Gran & $1.5 \%$ \\
\hline Sodium chloride & $25 \mathrm{~g} / 1$ \\
\hline Sodium carbonate & $6 \mathrm{~g} / 1$ \\
\hline Liquor ratio & $1: 10$ \\
\hline Dyeing temperature & $60 \square$ \\
\hline
\end{tabular}

\section{RESULTS AND DISCUSSION}

The residual analysis of pesticides in cotton by Agricultural Research Center, Central Laboratory of Residue analysis of pesticides.

Method name: Multiresidue method in cotton.

Method Description: Gas chromatographic multiresidue quantitative determination of organohalogen, organonitrogen,organophosphorus and some pyrithroids pesticide residues (Figure 2). 


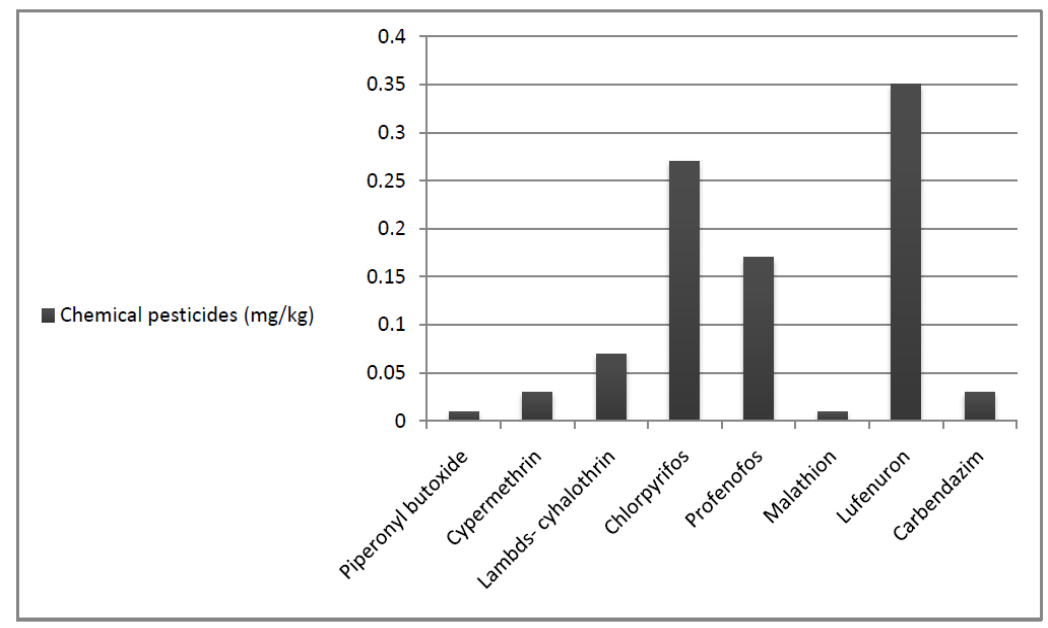

Figure 2. Residual analysis of pesticides in conventional cotton 
J. Environ. Sci.

Institute of Environmental Studies and Research - AinShamsUniversity

Table 4. Residue analysis of pesticides in conventional cotton

\begin{tabular}{|c|c|c|}
\hline Compound & Structure & Resultmg/kg \\
\hline $\begin{array}{c}\text { Piperonyl butoxide } \\
\text { 5-[2-(2 butoxyethoxy)ethoxymethyl]- } \\
\text { 6-propyl-1,3-benzodioxole }\end{array}$ & & 0.01 \\
\hline $\begin{array}{c}\text { Cypermethrin } \\
\text { [Cyano-(3-phenoxyphenyl)methyl]3- } \\
\text { (2,2-dichloroethenyl)-2,2- } \\
\text { dimethylcyclopropane-1-carboxylate }\end{array}$ & & 0.03 \\
\hline $\begin{array}{c}\text { Lambds- cyhalothrin } \\
\text { 3-(2-chloro-3,3,3-trifluoro-1- } \\
\text { propenyl)-2,2-dimethyl-cyano(3- } \\
\text { phenoxyphenyl)methyl } \\
\text { cyclopropanecarboxylate }\end{array}$ & & 0.07 \\
\hline $\begin{array}{c}\text { Chlorpyrifos } \\
\text { O,O-Diethyl O-3,5,6-trichloropyridin- } \\
\text { 2-yl phosphorothioate }\end{array}$ & & 0.27 \\
\hline $\begin{array}{c}\text { Profenofos } \\
\text { 4-bromo-2-chloro-1- } \\
\text { [ethoxy(propylsulfanyl)phosphoryl]ox } \\
\text { ybenzene }\end{array}$ & & 0.17 \\
\hline $\begin{array}{c}\text { Malathion } \\
\text { Diethyl 2- } \\
\text { [(dimethoxyphosphorothioyl)sulfanyl] } \\
\text { butanedioate } \\
\end{array}$ & & 0.01 \\
\hline $\begin{array}{l}\text { Lufenuron } \\
\text { 1-[2,5-Dichloro-4-(1,1,2,3,3,3- } \\
\text { hexafluoropropoxy)phenyl]-3-(2,6- } \\
\text { difluorobenzoyl) urea }\end{array}$ & & 0.35 \\
\hline $\begin{array}{c}\text { Carbendazim } \\
\text { Methyl 1H-benzimidazol-2-yl } \\
\text { carbamate }\end{array}$ & & 0.03 \\
\hline
\end{tabular}


Table 5 . residue analysis of pesticides in organic cotton

\begin{tabular}{|c|c|c|}
\hline Compound & Structure & $\begin{array}{c}\text { Result } \\
\mathbf{m g} / \mathbf{k g}\end{array}$ \\
\hline $\begin{array}{c}\text { Piperonyl butoxide } \\
\text { 5-[2-(2-butoxyethoxy)ethoxymethyl]- } \\
\text { 6-propyl-1,3-benzodioxole }\end{array}$ & & 0.01 \\
\hline
\end{tabular}

Table 6. Analytical results of textile wastewater.

\begin{tabular}{|c|c|c|c|c|c|c|}
\hline \multirow{2}{*}{ Process } & Type & $\begin{array}{c}\text { COD } \\
\text { (g O2/L) }\end{array}$ & $\begin{array}{c}\text { BOD } \\
\text { (g O2/L) }\end{array}$ & $\begin{array}{c}\text { TS } \\
(\mathbf{g} / \mathbf{L})\end{array}$ & $\begin{array}{c}\text { TDS } \\
(\mathbf{g} / \mathbf{L})\end{array}$ & pH \\
\hline \multirow{2}{*}{ Dyeing } & C. C & 3.8 & 1.6 & 14.8 & 0.9 & 10 \\
\cline { 2 - 7 } & O. C & 3.2 & 1.6 & 14.8 & 0.7 & 10 \\
\hline
\end{tabular}

\section{CONCLUSION}

The Conventional cotton has multiresidual chemical pesticides compounds ; Piperonyl butoxide , Cypermethrin, Lambds- cyhalothrin, Chlorpyrifos, Profenofos, Malathion, Lufenuron and Carbendazim. But organic cotton hasn't residual pesticides except Piperonyl butoxide . Analytical results of textile wastewater after wet processes and dyeing operations gave high BOD, COD, TS, alkaline wastewater and wasted residual unfixed dyes. But in case of conventional cotton is higher than organic cotton. There are no difference in colour fastness between conventional and organic cotton. Also there is no major difference in wet process and dyeing behavior of organic cotton when compared to conventional cotton except scouring loss, whiteness index, less heavy metal So organic cotton can be considered as Green processing of eco-friendly textiles with very low environmental impact and more healthy to human. 


\section{REFERENCES}

American Society for Testing Materials .ASTM. (1998): Standard test method of cotton fibers by High Volume Instrument (HVI), D-1776-98. Philadelphia 3, Pa, U.S.A.

American Society for Testing Materials (ASTM). (2004): Standard Atmosphere for Conditioning and Testing Designation, (D 177604).

Rieple A; Singh R. (2010): A value chain analysis of the organic cotton industry: The case of UK retailers and Indian suppliers, Ecological Economics, 69 : 2292-2302.

Couto S.R.( 2009): Dye removal by immobilised fungi. Biotechnology Advances; 27(3), 227-235.

Ferrigno,S. (2005): Pesticide action network UK 'Moral fibre: a beginner's guide to the UK market'. Available online at www.pan-uk.org/.

Madge, P. (1997): Ecological design: A new critique. Design Issues, 13 (2),44-54.

Punj, S.K. (2000): Plain knitted properties. Text. Asia. 31, 33-38.

Ton, P. (2002 :'Organic cotton production in sub-Saharan Africa: The need for scaling-up, A report for PAN UK's Pesticides Poverty and Livelihoods project, Pesticide Action Network UK (PAN UK), London, UK. 


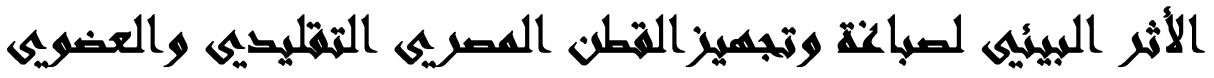

[r]

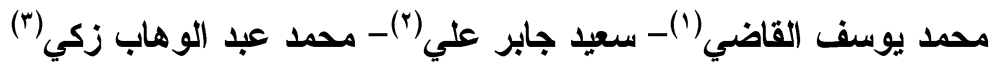

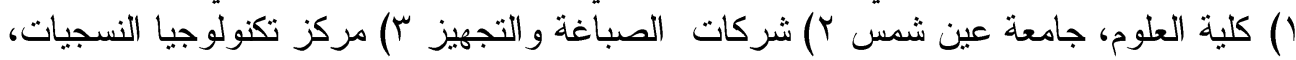

كلية الهندسة، جامعة القاهرة جلة المعة

\section{المستخلص}

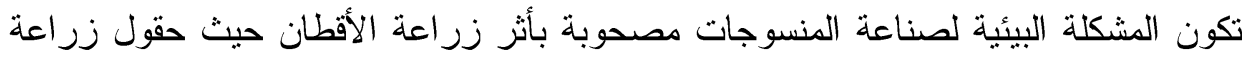

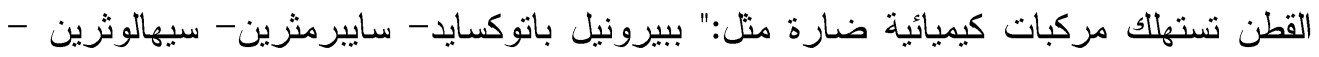

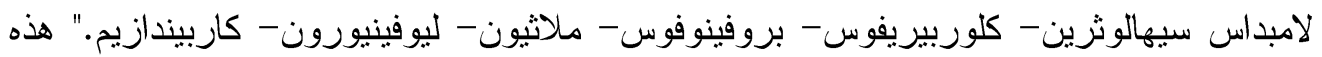

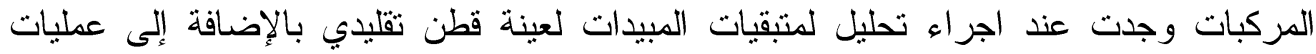

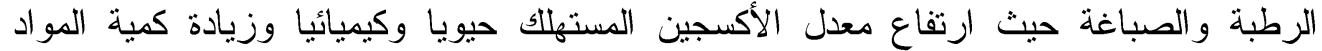

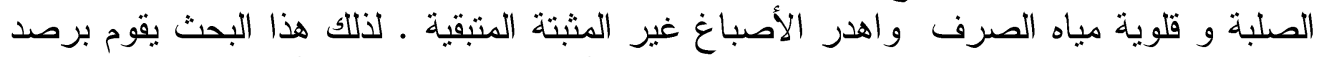

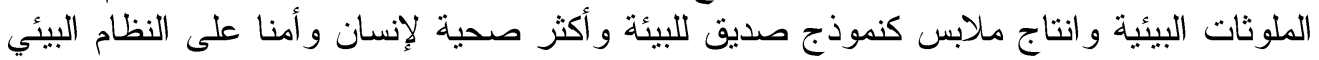

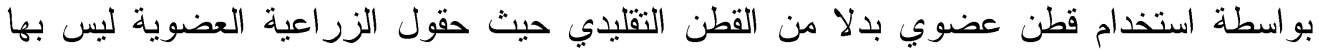

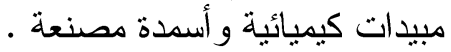

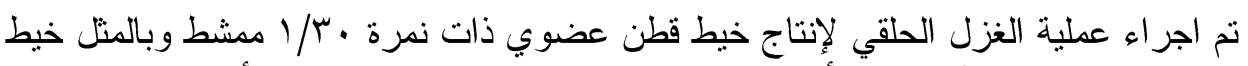

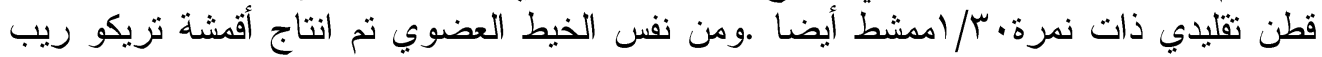

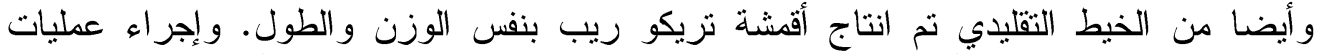

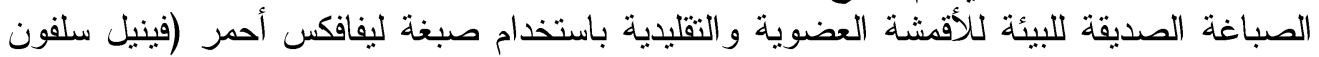

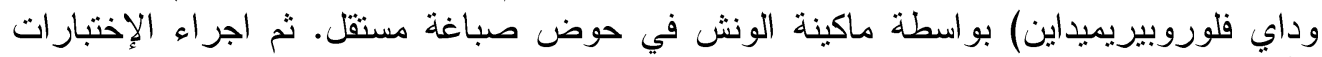

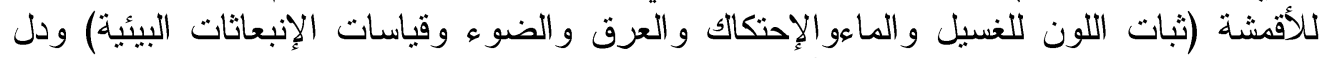
ذلك على انتاج ملابس صديقة للبيئة و أمنة على صحة الإحنس ولإنسان. 